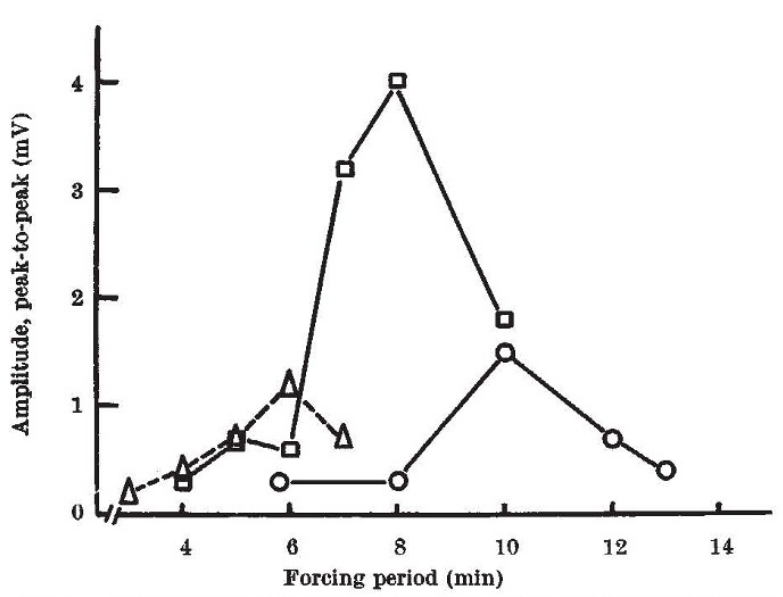

Fig. 2. Amplitude of electric oscillation of three different bean roots in response to $\pm 0.25^{\circ} \mathrm{C}$ oscillation of temperature at various periods. Mean temperatures: $0,15^{\circ} \mathrm{C} ;[], 17^{\circ} \mathrm{C} ; \triangle, 24^{\circ} \mathrm{C}$.

The roots also showed a resonant response to changes in the period of small oscillations of the temperature of the bathing medium (see Fig. 2). This behaviour is analogous to that found when osmotic pressure or con. centration of indolyl-3-acetic acid is made to oscillate ${ }^{3}$. The amplitude of the applied temperature oscillation was in the range $\pm 0 \cdot 15^{\circ} \mathrm{C}$ to $\pm 0 \cdot 25^{\circ} \mathrm{C}$; it was held constant within $0.02^{\circ} \mathrm{C}$ for each period. The resonant frequencies of some of the beans are shown as open circles in Fig. 1. The resonant frequency corresponds closely to the natural one. This lends further support to the feedback hypothesis, and suggests that the stimulus of small temperature oscillation does not unduly disturb the normal time constants of the feedback loop.

Jenkinson and Scott ${ }^{3}$ have shown that the difference in phase between forcing oscillation and bioelectric response provides information on the sequence of operations forming the loop. They have defined the quantity total phase shift as the amount by which this phase difference changes when the period of the forcing oscillation is changed from very short to very long values. The present work has yielded a total phase shift of about $90^{\circ} \mathrm{C}$ for temperature oscillations. This suggests that the temperature oscillation may be affecting the feedback system at a different point in the loop from that stimulated by oscillation of osmotic pressure $\left(270^{\circ}\right)$ or of indolyl-3-acetic acid $\left(180^{\circ}\right)$.

Future work involves a more detailed investigation of the phase relationships, and of the effect of metabolic inhibitors, with a view to providing a clearer understanding of the nature of the feedback system.

This work was supported by a U.S. Public Health Service research grant from the National Institutes of Health.

R. L. GUNTHER

B. I. H. SootT

Biophysics Laboratory,

Department of Physics,

University of Tasmania,

Hobart.

McAulay, A. L., and Scott, B. I. H., Nature, 174, 924 (1954).

2 Scott, B. I. H., A ustral. J. Biol. Sci., 10, 164 (1957).

${ }^{3}$ Jenkinson, I. S., and Scott, B. I. H., Austral. J. Biol. Sci., 14, 231 (1061).

4 Scott, B. I. H., Ann. N.Y. Acad. Sci., 98, 890 (1962).

'Jenkinson, I. S., A Austral. J. Biol. Sci, 15, 115 (1962).

\section{A Possible Explanation for the Effect of Magnetic Fields on Biological Systems}

Althougr there is considerable controversy over the validity of any given example of the influence of a magnetic field on a biological system, the number of such reported phenomena ${ }^{1}$ continue to make it a useful task to speculate on mechanisms which are reasonable on a molecular level. The direct influence of magnetic fields on chemical equilibrium or rate of reaction has occasionally been reported; however, this cannot usually be reproduced, and the general weight of evidence is that such effects are insignificant ${ }^{2}$. No satisfactory picture of what a weak magnetic field can do to molecular-level phenomena seems to have been advanced.

To the best of our knowledge the explanation offered here has been overlooked, and appears to offer a consistent and reasoned basis for suggesting that a magnetic field, of the order of 1,000 gauss $(G)$ or more, influences the phenomena of charge transport, both ionic and electronic, mass transport (diffusion rate), and reaction rate in a biological medium. The argument is based on the combination of two rather well-known and well-documented observations: (1) the rod-like molecules in a liquid crystal can orient themselves in a magnetic field; (2) liquid crystalline materials have been identified in a host of biological systems.

Reports on liquid crystals $^{3}$ have included several examples of the influence of weak magnetic fields on the orientation of the rod-like molecules and of the consequences of this phenomenon. Forty years ago, using relatively weak magnetic fields $(1,000-5,000 \mathrm{G})$, Svedberg investigated the rates of reactions carried out in mesomorphic states as solvents ${ }^{4}$. He studied the unimolecular thermal decomposition of picric acid, pyrogallol and trinitroresorcinol in $p$-azoxyphenetole, and found that the rate decreased significantly when the liquid crystal was oriented in an external magnetic field. Svedberg also studied rates of diffusion of $m$-nitrophenol in a mixed liquid crystal system of $p$-azoxyanisole and $p$-azoxyphenetole ${ }^{5}$. He found that magnetic fields affected the rate of diffusion-the rate rose when the magnetic fields were parallel to the direction of flow, and fell when the field was applied across the direction of flow.

There have been many reports of the effect of magnetic fields on the dielectric properties of liquid crystals ${ }^{3}$. For example, Maier, Barth and Wiehl ${ }^{6}$ found that the difference in the transverse and the longitudinal dielectric constant of 4,4' -di- $p$-methoxyazobenzene increases rapidly up to $1,000 \mathrm{G}$, and is then independent of field strength. The picture which has developed is that the rod-like molecules, or ellipsoidal swarms of these molecules, are oriented by magnetic fields of the order of 1,000 G and that many of their properties are markedly influenced.

That biological systems are replete with liquid crystalline material has also frequently been reported. For example, Stewart ${ }^{7}$ showed that the complex lipids present in the adrenal cortex, ovaries and myelin exist at body temperature in a characteristic mesophase. Robinson ${ }^{8}$ has shown a widespread occurrence of the cholesteric phase in polypeptide solutions and biological structures. Reviews of much of this work were presented at the 1965 International Liquid Crystal Conference?

This explanation might apply to both "field sensitive" and "gradient sensitive" phenomena". The orientation of rods parallel to a magnetic field occurs for both diamagnetic and paramagnetic materials ${ }^{10}$.

Chemistry Division,

M. M. LABES

The Franklin Institute Research Laboratories, Philadelphia.

${ }^{1}$ See, for example, Biological Effects of Magnetic Fields, edit. by Barnothy, M. F. (Plenum Press, New York, 1964).

"Mulay, I. L., and Mulay, L. N., in Biological Effects of Magnetic Fields, edit. by Barnothy, M. F., 163 (Plenum Press, New York, 1964).

Brown, G. H., and Shaw, W. G., Chem. Rev., 57, 1049 (1957). Gray, G. W. Molecular Structure and the Properties of Liquid Crystals (Academic Press, New York, 1962).

‘ Svedberg, T., Kolloid Z., 18, 54, 101 (1916); 21, 19 (1917).

s Svedberg, $T$, Kolloid $Z$, 22, 68 (1918).

- Maier, W., Barth, G., and Wiehl, H. E., Z. Electrochem., 58, 674 (1954).

'Stewart, G. T., Nature, 183, 873 (1959).

${ }^{8}$ Robinson, C., Trans. Farad. Soe., 68, 571 (1956).

Intern. Liquid Crystal Conf., Kent ,State Oniv., Kent, Ohio, August 10-20, 1965, Part I, Molecular Crystals, 1, 201 (1966).

${ }^{10}$ Bates, I. F., Modern Magnetism, 95 (Cambridge Univ. Press, 1939). 